\title{
UV SPECTROPHOTOMETRY OF CANOPUS FROM GEMINI XI
}

\author{
Y. KONDO, K. G. HENIZE*, and C. L. KOTILA \\ Astronomy Branch (TG4), NASA Manned Spacecraft Center, Houston, Texas, U.S.A.
}

\begin{abstract}
The UV energy curve of Canopus in the $2400-4000 \AA$ wavelength range has been obtained from spectrograms having a dispersion of $183 \AA \mathrm{mm}^{-1}$. This curve is in good agreement with previous observations and with a model atmosphere. The equivalent width of $\mathrm{Mg}_{11} 2795 \AA, 2802 \AA$ is found to be $22 \AA$.
\end{abstract}

Objective-grating spectra of the F0 supergiant Canopus ( $\alpha$ Car) were obtained by astronauts C. Conrad and R. Gordon during the 2-hour extravehicular activity on the Gemini XI manned space flight on September 14, 1966. The equipment and operating procedures have been described in detail by Henize et al. (1968). This paper reports the results of the photometric reduction of the UV spectrum of Canopus. A more detailed paper will be published elsewhere (Kondo et al., 1970).

Figure 1 illustrates the best spectrum obtained and identifies the prominent absorption features. A discussion of these identifications has been given by Henize et al. (1967). The dispersion of the original spectrum is $183 \AA \mathrm{mm}$ and the resolution, estimated from the diameters of the zero-order images, is approximately $15 \AA$.

Figure 2 shows the composite energy curve derived from measures of the bestexposed regions of three spectra. The straight lines drawn in this figure represent our estimate of the position of the continuous spectrum unaffected by obvious selective absorption features. This estimated continuum is used for comparison with other data in Figure 3, in which our data are compared with a model atmosphere by Parsons and with observational data by Aller et al. (1966) and by Stecher (1969). Aller's data and the model atmosphere were fitted to our data at $4000 \AA$ while Stecher's data were fitted to ours at $3000 \AA$. The model atmosphere data were kindly supplied by Parsons especially for this comparison using methods described in a previous paper (Parsons, 1969). The chosen parameters were $T_{e}=6900 \mathrm{~K}, \log g=2.0$ for a non-LTE, blanketed model.

In general, the Gemini XI data appear to be in good agreement with both the previous observations and the model atmosphere. Our measured value for the magnitude difference between $3640 \AA$ and $4000 \AA$ (1.32 mag) is in excellent agreement with the model atmosphere. The slight disagreement with Aller's data approximates the expected probable error of our photometry. The fit with Stecher's data is good if we note that Stecher has not interpolated the continuum across the large absorption complex from $2600 \AA$ to $2900 \AA$ as we have. This agreement tends to confirm Stecher's data and suggests that the anomalous behavior of his observed continuum in the 2000-2300 $\AA$ region deserves further investigation. The only significant dis-

* On leave of absence from Lindheimer Astronomical Research Center, Northwestern University, Ill., U.S.A. 


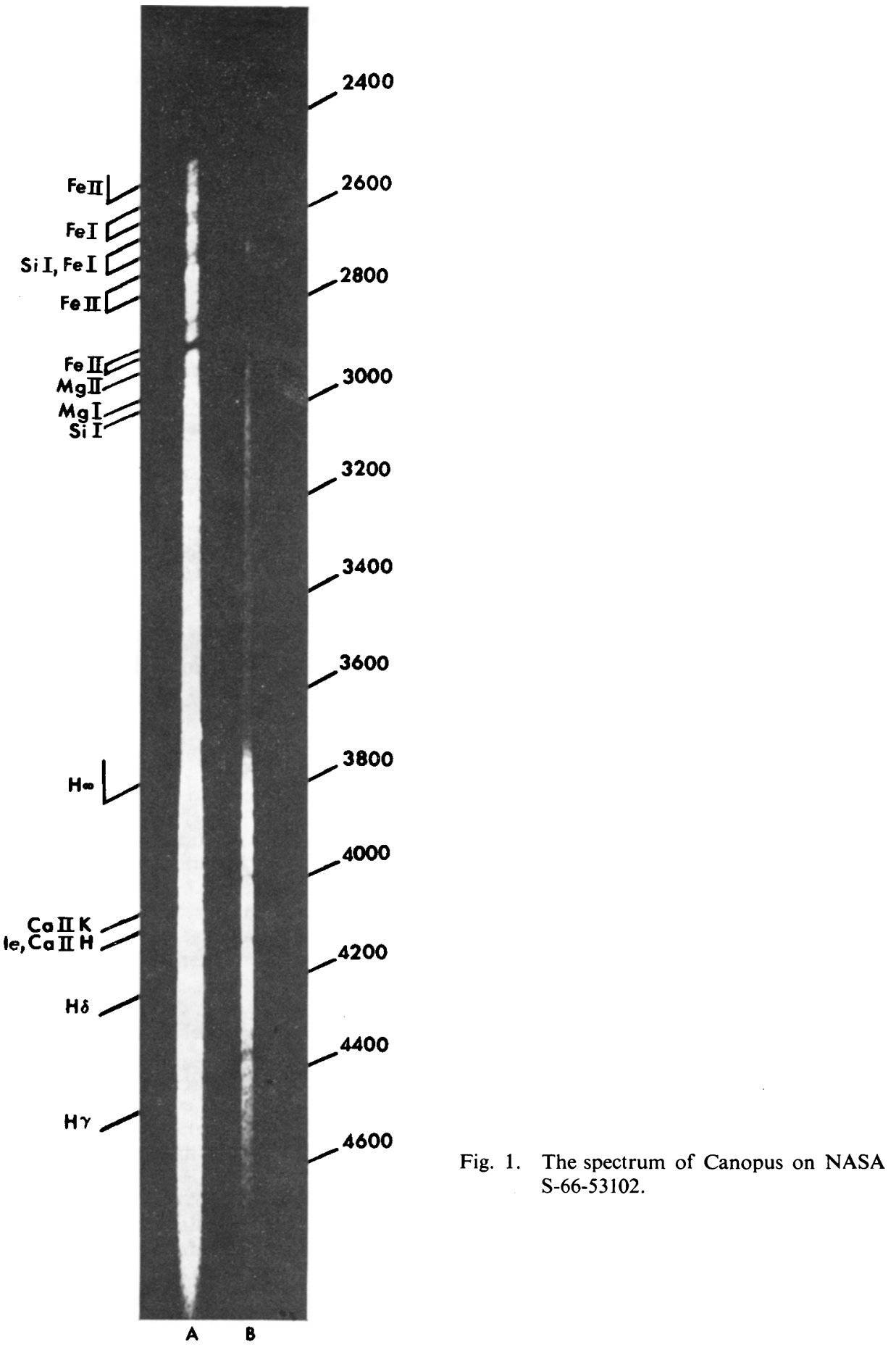




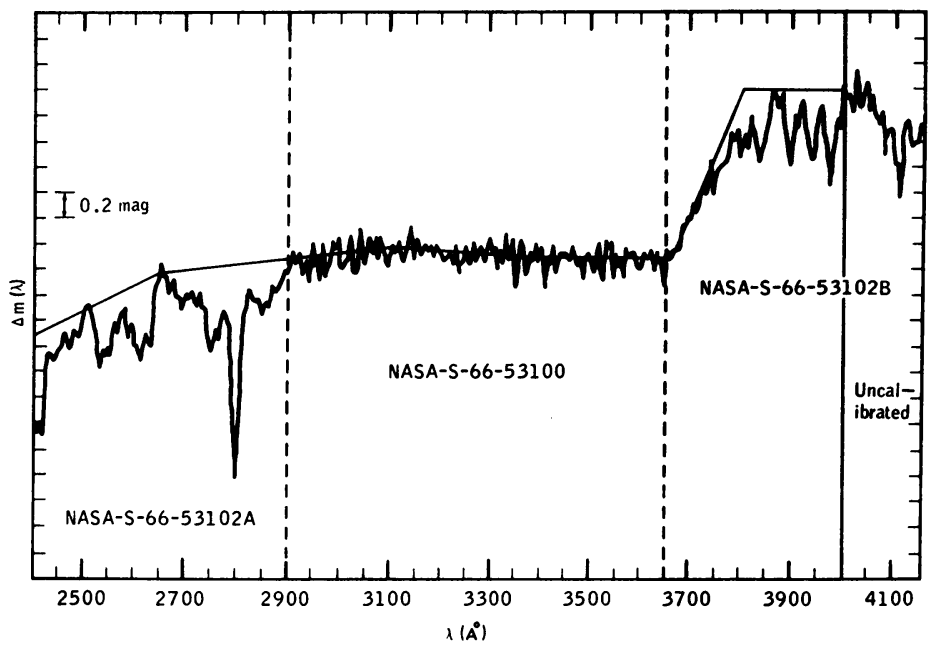

Fig. 2. UV spectral energy curve of Canopus.

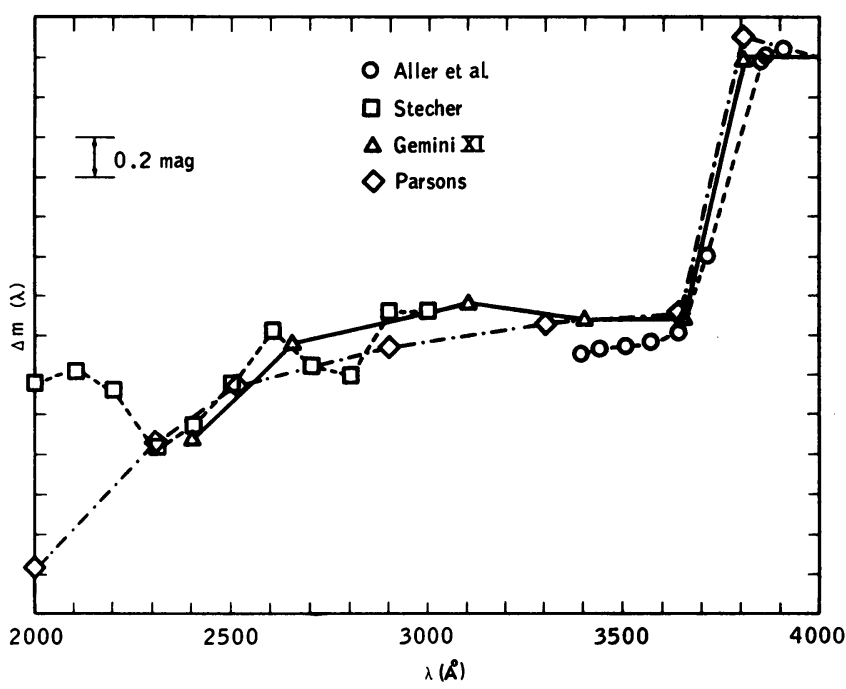

Fig. 3. Comparison of UV observations of Canopus and a model atmosphere.

TABLE I

Equivalent widths in the UV spectrum of Canopus

\begin{tabular}{llcl}
\hline Wavelength $(\AA)$ & Ident. & $W(\AA)$ & Remarks \\
\hline $2520-2560$ & blend of Fe I & 15 & Possibly includes SiI resonance lines \\
$2580-2640$ & blend of Fe II & 22 & Possibly includes MnII resonance lines \\
$2735-2775$ & blend of Fe II & 18 & \\
2799 & $\mathrm{Mg}_{\text {II }}$ & 22 & Resonance doublet 2795 $\AA, 2802 \AA$ \\
2852 & $\mathrm{Mg}_{\text {I }}$ & 3.4 & Resonance line \\
2882 & $\mathrm{Si} \mathrm{I}$ & 2.0 & Very weak, perhaps doubtful
\end{tabular}


crepancy between our data and the model atmosphere is the elevation of our continuum above that of the theoretical continuum in the $2550-3400 \AA$ region. Although it is not impossible that this may be a result of inaccuracies in our calibration it seems probable that a real discrepancy with the model may exist.

Measures of equivalent widths, made on direct intensity plots using a continuum closely approximating that shown in Figure 2, are given in Table I. Blended features are included since it is probable that their strengths will be of interest in UV classification schemes. For example the two broad absorption features extending from 2520 to $2640 \AA$ and from 2740 to $2900 \AA$ are visible in Gemini UV objective-prism spectra having a dispersion of $1500 \AA \mathrm{mm}^{-1}$ at $2500 \AA$. The most significant measure is that of the MgII doublet, since this is the resonance line of that ion and is of sufficient strength to be measurable over a broad range of spectral classes. Its equivalent width given in Table I should be taken as a lower limit since the area of the line was taken as a simple triangle and no allowance was made for the possible existence of broad wings.

\section{Acknowledgements}

We wish to thank Roy C. Stokes, NASA Manned Spacecraft Center, and F. G. O'Callaghan, Northwestern University, for their contributions to this work. Stokes was responsible for the development of the spectrograph and participated in the data reduction. O'Callaghan was primarily responsible for the film calibration system. This work was supported by NASA contract NAS 9-4129 to Northwestern University.

\section{References}

Aller, L. H., Faulkner, D. J., and Norton, R. H.: 1966, Astrophys. J. 144, 1073.

Henize, K. G., Wackerling, L. R., and O'Callaghan, F. G.: 1967, Science 155, 1407.

Henize, K. G., Wray, J. D., and Wackerling, L. R.: 1968, Bull. Astron. Inst. Czech. 19, 279.

Kondo, Y., Henize, K. G., and Kotila, C. L.: 1970, Astrophys. J. 159.

Parsons, S. B.: 1969, Astrophys. J. Suppl.. Ser. 18, 127.

Stecher, T. P.: 1969, Astron. J. 74, 98.

\section{Discussion}

Bonnet: It seems that your spectrum looks very much like the solar spectrum in the vicinity of the $\mathrm{Mg}$ I discontinuity due to photo-ionization from the first excited level of $\mathrm{Mg}$. More particularly there is a difference between the intensities of the two peaks appearing in your spectrum at $2500 \AA$ and $2650 \AA$. Do you think that this difference might be due to $\mathrm{Mg}_{\mathrm{I}}$ ionization as it is the solar spectrum?

Henize: I have not considered the possible presence of the $\mathrm{Mg}$ I discontinuity. I believe that the obvious absorption features in this region of the spectrum are adequately accounted for by blended iron lines but it is possible that the $\mathrm{Mg}$ I discontinuity contributes to the feature extending shortward from $2500 \AA$.

Stecher: It is nice to be on the high side of theory for a change. The points are, of course, very weak at the short wavelengths and are dependent upon the background level.

Henize: Please pardon me for bringing undue attention to the disagreement between your data and the model. However, I was quite pleased by the agreement between your data and ours in our 
region of overlap and I feel that the discrepancy with theory suggested by your shorter wavelength data deserves further investigation.

Conti: (1) What was the exposure time for the Canopus spectrum? (2) What plans are underway to continue these spectrographic observations in future Apollo programmes?

Henize: (1) The longest exposure was 2 min. (2) At Northwestern University, we have designed and built a 6-inch aperture objective prism spectrograph which is scheduled to be flown on the first Apollo Applications Programmes flight. A calcium fluoride prism is the dispersing element. We expect to record spectra down to $1350 \AA$ with a resolution of about $1 \AA$ at $1500 \AA$. 